\title{
Kocharyan A.M. \\ Customer support system of a multifunctional public services center based on machine learning
}

Amur State University

(Russia, Blagoveshchensk)

doi: $10.18411 /$ satm-02-2022-02

\section{Abstract}

Aspects of the development of a chatbot for working with citizens' appeals are considered, which helps to reduce the burden on call center operators and consultations with clients on the same type of requests, the number of which is constantly growing. The ideas of taking into account the variability of the scenario of consultations on the order of presentation of public services and identifying new trends in the requests of applicants are presented.

Keywords: NLP, question-and-answer system, chatbot.

According to the Ministry of Economic Development of the Russian Federation, the activity of electronic requests for public services increased by 500 thousand per week in July 2020 . According to the department, citizens' requests are predominantly of the same type, so it is possible to automate the formation of a user scenario of service provision, as well as to identify scenarios for the direction of chatbot development.

There are many chatbot builders' platforms in the market such as "Botmother", "Aimylogic", "Dialog Flow Framework", which provide tools for creating bots. However, these solutions have significant drawbacks, expressed as a lack of flexibility, ready-made integrations with business tools, functionality for understanding natural language, or high cost.

One of the key ideas for developing a customer support system is to use current research on systems with natural language interfaces. For example, a system architecture with a natural language interface containing a search and filter module can be developed to answer the same type of frequently asked questions. The search module has built-in statistics for ranking the relevance of the document in the corpus, taking the client's request into account:

$$
t f(t, d)=\frac{t f_{-} i d f(t, d, D)=t f(t, d) \cdot i d f(t, D),}{\sum_{t^{\prime} \in d} f_{t^{\prime}, d}}, \quad i d f(t, D)=\log \frac{N}{|\{d \in D: t \in d\}|},
$$

where $\mathrm{tf}$ - term frequency, idf - inverse document frequency,

$N$ - number of documents in the corpus, $t$ - term, $d$ - document,

$f_{t, d}$ - number of terms in the document.

The "term frequency - inverse document frequency" measure has been presented as one of the ways to transform textual information into vector-spatial model. Therefore, the similarity metric for vector representation of documents - the cosine similarity - was used as a filter:

$$
\text { cosine similarity }=\frac{\sum_{i=1}^{n} A_{i} B_{i}}{\sqrt{\sum_{i=1}^{n} A_{i}^{2}} \sqrt{\sum_{i=1}^{n} B_{i}^{2}}},
$$

where $A_{i}$ and $B_{i}$ are components of vectors $A$ and $B$, respectively.

Such an architecture allows classifying an incoming user's question and providing an answer to the most similar question, and represents a kind of "intention detector". When an incoming question is very different from those in the database, which can be determined based on the cosine similarity value, it is added to the database and the responsibility for providing the service is transferred to the employee of the organization. In this way, the direction of the bot's development can be determined.

Figure 1 shows an example of the model, trained on the "question-answer" data set, collected from the data from the website of the Multifunctional Public Services Center of the Amur region. 


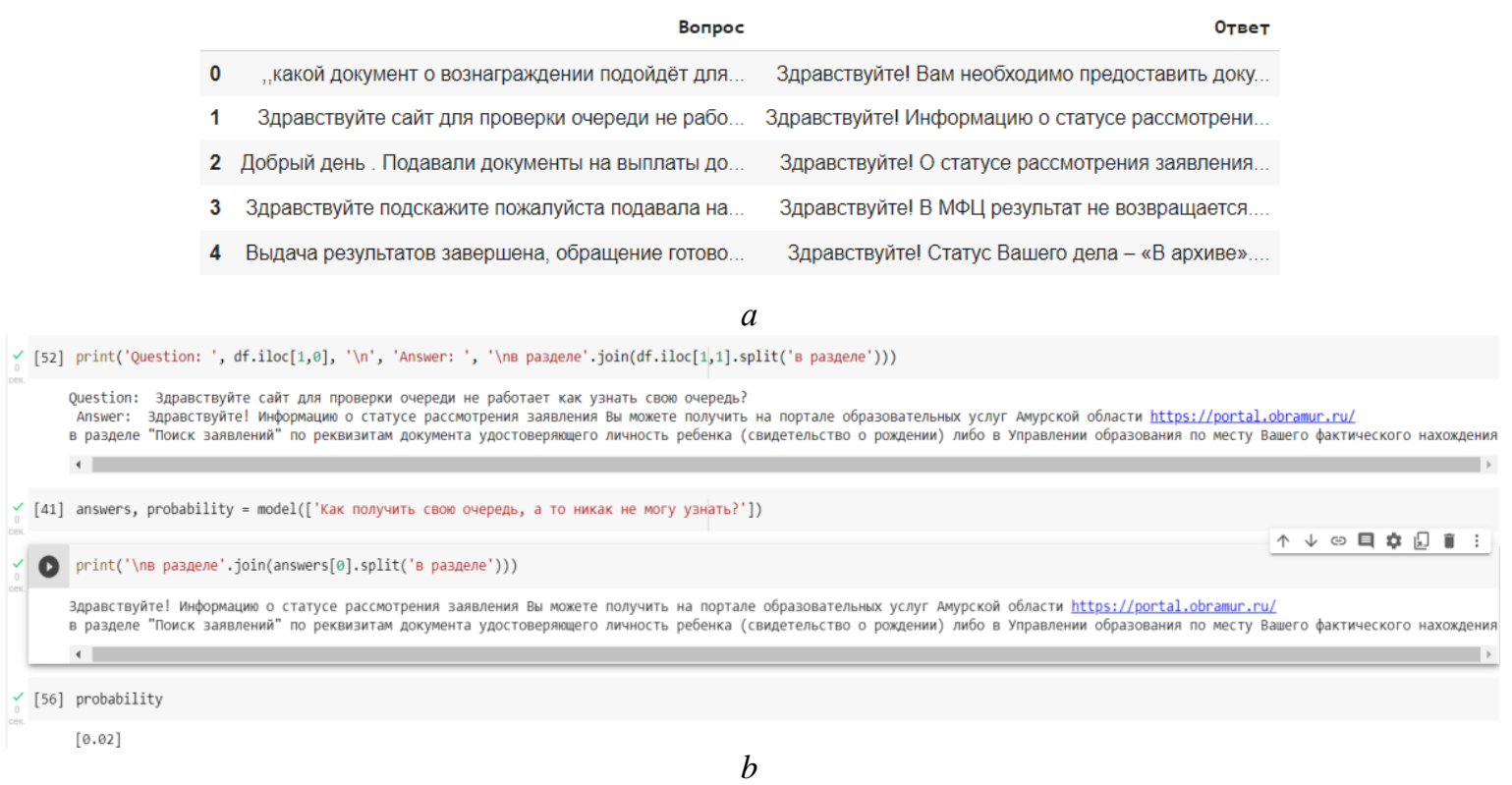

Figure 1. The functioning of the question-answering model on the data of the Multifunctional Public Services Center of the Amur region

By empirically setting the value of the cosine similarity threshold, it is possible to control the flow of program execution. A way to obtain the necessary information from the news and social media posts of an organization was proposed: if the answer is not found in the database (the cosine similarity value is small), then the control is transferred to a special module, which extracts the answer from the text information, which stores the actual news of the organization. Such a task is reduced to the SQuAD research problem. According to the published results of the deeppavlov NLP library development team, which can be found at https://deeppavlov.ai, the results of the latest competitive solutions reach 88.49 on the F1 metric, which provides a high-quality solution.

The implementation of a multifunctional public services center chatbot based on deep learning and natural language processing technologies as a customer support system allows reducing the load on the call center operators, improving their quality of work without the need to hire additional staff, increasing the speed of service delivery.

$* * *$

1. Rybak, K.V., Koshkarov, A.V. (2020). Review of the current state of intelligent question answering systems. International Scientific Journal "Vestnik Nauki", 6, 202-205.

2. Naumenko, A.M., Sheludko, S.D., Yuldashev, R.Y., Khlebnitsky, N.O. (2017). Development of question answering system using machine learning. International Scientific Journal "Molodoi Uchenyi", 36-39.

3. DeepPavlov Library documentation. Retrieved August 27, $2021 \quad$ from http://docs.deeppavlov.ai/en/master/index.html. 